\title{
Size Effect Analysis of Scale Test Model for High-speed Railway Foundation under Dynamic Loading Condition
}

\author{
Shuren WANG*, Kunpeng SHI, Youfeng ZOU, Zhengsheng ZOU, Xuchun WANG, Chunliu LI
}

\begin{abstract}
To determine the energy attenuation and energy reflection coefficients in layered foundation is the key factor to reveal the dynamic response characteristics of the high-speed railway foundation. Based on the foundation test model under the dynamic loading of the high-speed railway, the energy attenuation and energy reflection coefficients were introduced and the attenuation formulas of the vibration acceleration in the layered foundation were deduced. Five scale models of 1:1, 1:2, 1:5, 1:10 and 1:20 are established respectively by using Abaqus technique. The energy attenuation mechanism and interfacial energy reflection characteristics in the layered foundation were analyzed. Results show that it is appropriate to use vibration acceleration to characterize the propagation rule of the energy attenuation in the layered foundation. The size effect equations of the energy attenuation and energy reflection coefficients in the layered foundation are deduced and the size effect of the energy attenuation is revealed. Based on the nonlinear relationships among the energy attenuation coefficient, the model scale, the loading amplitude and the vibration frequency, the energy attenuation equation of the scale test model is constructed. The reliability of the theoretical and simulation results is verified by the scale test model. The conclusions obtained in this study can provide a reference for a similar engineering practice.
\end{abstract}

Keywords: high-speed railway foundation; ize effect; senergy attenuation; vibration acceleration

\section{INTRODUCTION}

The high-speed railway foundation will produce dynamic stress and vibration acceleration due to the action of the vibration load during the high-speed railway operation. To investigate the dynamic response characteristics of the high-speed railway foundation, some scholars have conducted many research works on the related field by the field monitoring, indoor tests, and numerical analysis [1-3].

Due to the time-consuming and expensive field test, the indoor test has become the main research method for the dynamic response of the high-speed railway foundation under dynamic loading condition. Since the physical and mechanical properties between the scale test model and the prototype test cannot fully meet the similarity ratios, the size effect is often caused by a deviation between these test results [4]. Although there are many results about this topic, it is mostly still in empirical exploration or case studies [5-10].

Determining the energy attenuation and energy reflection coefficients in layered foundation is the key to reveal the dynamic response characteristics for high-speed railway foundation. In order to reveal the energy attenuation mechanism and interfacial energy reflection characteristics in the layered foundation, it is of important meaning to conduct the size effect analysis of the scale test model for the high-speed railway foundation under the dynamic loading condition.

\section{STATE OF THE ART}

Previous studies on the size effect are mostly focused on model materials, such as the sizes of concrete and asphalt aggregate particles [11-13]. However, there were a few studies on the size effect of the model or structure. Knox et al. established the laboratory test models of masonry structures with two scales, and they found that the model size had no influence on the compressive stiffness of masonry, while the diagonal shear strength and shear modulus had significant influences [14].
Pirooznia \& Moradloo found that the relative defects and attenuation of the dispersed fracture model could effectively describe the size effect [15]. Gao et al. used a failure model to express the size and boundary effects and they established the dimensional effect prediction model of the geometrically similar components of the concrete curved beam [16]. Alamet al. proposed the size effect model and made it possible to determine the size independent fracture properties [17]. Chen et al. studied the dimensional effect on the load-settlement response of the foundation of a homogeneous sand layer [18].

According to the similarity ratio, it is extremely difficult for the scale test model to achieve similar materials, external loads, and stress-strain that are exactly consistent with the prototype test. Most scholars usually use one or two physical quantities to characterize the size effect of the scale test model. Jin et al. constructed a threedimension numerical method to simulate the destruction of the reinforced concrete columns based on the classical dimensional effect theory of the concrete materials [19]. From the perspective of pile impedance and foundation settlement, Liu et al. studied the size effect of the rigid pile composite foundation [20]. Yoder et al. found that the dimensional effect of porous material was related to the bending strain energy [21]. Lee \& Han studied the size effect of the foundation, and they found that the bearing capacity coefficient decreased with increase of the foundation width [22]. Vishwakarma \& Ingle found that the critical stress was higher for the smaller concrete pavement panel sizes [23]. Mehrjardi \& Khazaei determined that the size effect of the reinforced soil played an important role in the response of the reinforced foundation [24].

The dynamic size effect of the scale test model was different from the static size effect [25-31]. Lee et al. conducted the dynamic and static tests on different cylinder sizes and they found that the size effect became substantial in high strength concrete greater than $40 \mathrm{MPa}$ [32]. To further study the dynamic size effect of the roller compacted concrete, Wang et al. studied many roller compacted concrete specimens with different sizes under 
the impact loadings, and they proposed an effective way to evaluate the dynamic behaviour of the roller compacted concrete based on the fragment size distribution [33]. Most of the abovementioned studies are from the aspects of the compression, bending, shear strength and the foundation settlement of the specimens or components. However, there are few studies on the dynamic size effect of the indoor test models. In practical engineering, the mechanical condition of the test model is very complicated under dynamic load. It is important to study the size effect of the indoor test model from the perspective of the dynamics to understand the dynamic properties of the scale test model.

Based on the energy attenuation principle of the dynamic loading in the high-speed railway foundation, this study used the vibration acceleration as a variable to comprehensively represent the energy attenuation of the foundation. Five geometric similarity ratio models (1:1, $1: 2,1: 5,1: 10$, and $1: 20)$ were established to analyze the internal relationship among the energy attenuation and the interface energy reflection coefficients of the layered soil and the size of the foundation model. The corresponding size effect equation was constructed. The influences of the loading amplitude and vibration frequency on the model size effect were studied by indoor model tests. The research results can provide the theoretical basis for the application of scale test model, and provide a reference for the similar engineering practice.

The remainder of this study is organized as follows. In Section 3, the energy attenuation formula is derived, which is verified by numerical simulation results. The similarity principle in the scale test model is stated and the computational model is built. In Section 4, the energy attenuation and size effect are analyzed and discussed. Finally, some conclusions are given in Section 5.

\section{METHODOLOGY}

\subsection{Energy Attenuation Formula and Its Verification 3.1.1 Energy Attenuation Formula in Homogeneous Soil}

According to the wave attenuation theory, due to the influences of the geometric and material dampings of the medium, the energy of the elastic wave propagation in the medium will attenuate.The energy attenuation rule of the stress wave propagating along the depth direction in the soil is

$$
I=I_{0} \mathrm{e}^{-\alpha H}
$$

where, $I$ is the wave strength in the depth $H$ of the foundation. $I_{0}$ is the initial wave strength. $\alpha$ is the wave attenuation coefficient, which is related to the wave frequency and the soil properties.

The wave strength $I$ at a certain point in the foundation soil changes periodically according to the sine function, and the stress amplitude is

$$
I=\rho \omega^{2} D^{2}
$$

where, $\rho$ is the density of the foundation soil, $w$ is the round frequency, $D$ is the vibration amplitude.
The spatial position relationship of the vibration load of the high-speed railway, subgrade and foundation is shown in Fig. 1.

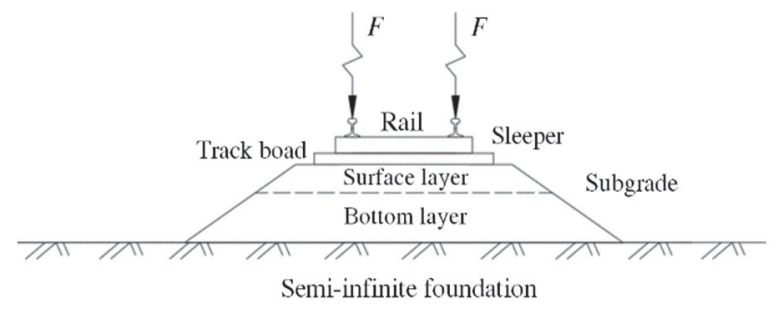

Figure 1 Schematic diagram of the high-speed railway load, subgrade and foundation

According to China Foundation Dynamic Characteristic Test Specification (GBT50269-2015), the amplitude at a certain point in the foundation also changes periodically as a sine function, which attenuates away from the vibration source in the form of a negative exponential in the soil. Since the relationship between the displacement and acceleration is two derivatives. therefore, the relationship between the vibration acceleration and amplitude can be obtained by differentiating the amplitude sinusoidal periodic function twice:

$a=\omega^{2} D$

where, $a$ is the vibration acceleration.

The intensity of the stress wave in Eq. (2) is proportional to the square of the amplitude, while the amplitude in Eq. (3) is proportional to the vibration acceleration. Combining Eqs. (2) and (3), the relationship between the intensity and the vibration acceleration of the stress wave is

$$
I=\rho \omega^{2}\left(\frac{a_{\max }}{\omega^{2}}\right)^{2}=\rho \frac{a_{\max }^{2}}{\omega^{2}}
$$

Combining Eqs. (1) and (4), then Eq.(5) can be obtained as:

$$
a=\omega \sqrt{\frac{I_{0}}{\rho}} \mathrm{e}^{-\frac{\alpha H}{2}}
$$

During the propagation of stress waves generated by a fixed vibration source, $I_{0}, \omega, \rho$ are constants, respectively. Let $\omega \sqrt{\frac{I_{0}}{\rho}}=a_{0}$, where, $a_{0}$ is the initial vibration acceleration of the vibration source; and let $\alpha / 2=\beta$, where $\beta$ is the attenuation coefficient of the vibration acceleration. Eq. (5) can be simplified as:

$$
a=a_{0} \mathrm{e}^{-\beta f H}
$$

where, $f$ is the frequency.

Eq. (6) shows that the transmission of the vibration acceleration along the depth direction of the foundation 
presents an exponential attenuation, which is consistent with the attenuation rule of the stress wave and amplitude.

Simultaneously, from the perspective of theoretical formulas, the dynamic strain of the foundation soil caused by the high-speed train loading in the middle and early stages of subgrade operation is very small, and the foundation generally belongs to the elastic deformation stage. Thus, the stress waves produced by the dynamic load in the foundation soil can be approximately regarded as elastic waves, so it can be concluded that:

$$
\sigma_{d}=\frac{D}{H} E_{\mathrm{d}}
$$

where, $\sigma_{\mathrm{d}}$ is the dynamic stress in the soil and $E_{\mathrm{d}}$ is the dynamic elastic modulus.

From Eq. (3), we can obtain

$$
\sigma_{\mathrm{d}}=\frac{a}{H \omega^{2}} E_{\mathrm{d}}
$$

Eqs. (3) and (8) show that there is an internal relationship between the vibration acceleration and the dynamic stress. Therefore, it is appropriate to use the propagation of the vibration acceleration in the foundation to characterize the attenuation of energy waves.

\subsubsection{Energy Transfer Law at Interface of Soil Layers}

In actual engineering, the foundation is complex and mostly multilayered soil. Since the energy wave impedances of different types of soil are different, it is similar to the different laws where the light propagates in different media. The energy wave follows different attenuation rules in different soils. When the vibration load is transmitted to the soil interface in the form of an incident stress wave, it will produce the refracted wave and reflected stress waves. Based on the principle of conservation of energy, the incident wave equals the reflected wave plus the transmitted wave, and the energy wave passing through the soil interface is bound to weaken, as shown in Fig. 2.
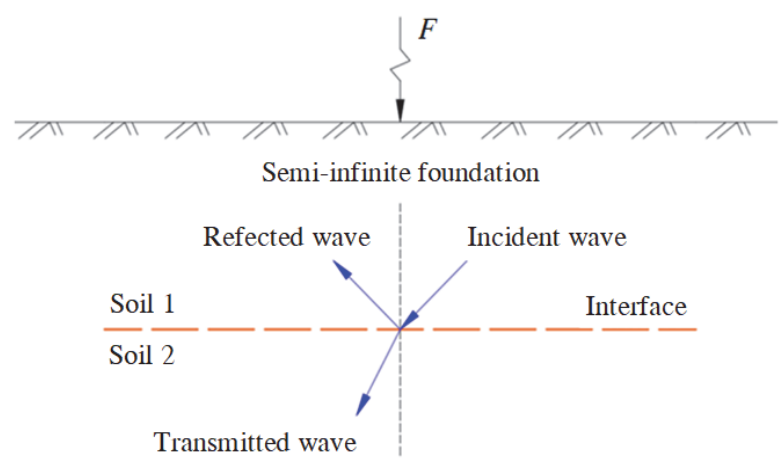

Figure 2 Schematic diagram of the vibration propagation and reflection in a semi-infinite foundation

In the layered foundation, the energy wave transmission in different soil layers follows the attenuation law of the corresponding soil types, as shown in Fig. 3. The energy reflection coefficient of the soil layer is introduced as:

$$
\gamma=\frac{a_{1}-a_{2}}{a_{1}}=\frac{\Delta a}{a_{1}}
$$

where, $a_{1}$ is the vibration acceleration of the upper soil at the soil interface, and $a_{2}$ is the vibration acceleration of the lower soil at the soil interface. In Fig. 2, $a_{1}$ and $a_{2}$ correspond to the incident wave and the refracted wave, respectively, and $\Delta a$ corresponds to the reflected wave.

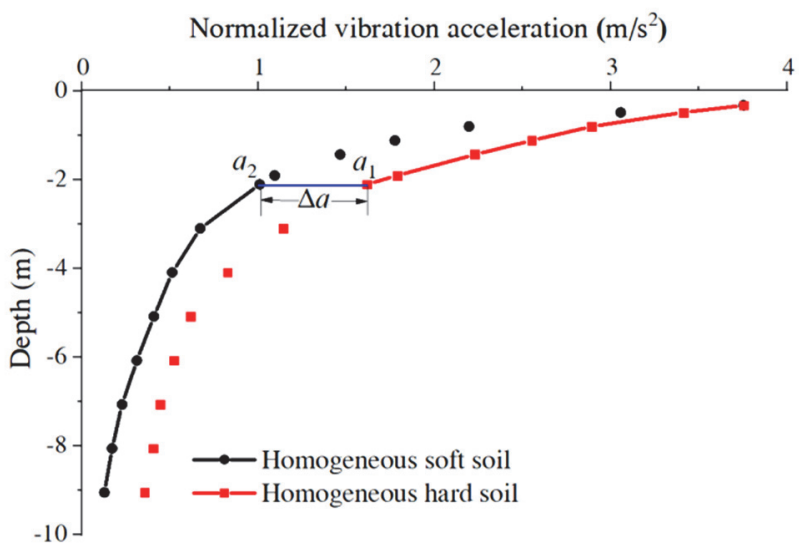

Figure 3 Attenuation law of vibration acceleration of the layered foundation

Substituting Eq. (6) into Eq. (9),

$$
\gamma=1-\mathrm{e}^{-H f\left(\beta_{2}-\beta_{1}\right)}
$$

where, $H$ is the interface depth of the soil layer.

Eq. (10) shows that the energy reflection coefficient is not only related to the energy attenuation coefficient but also has the relationship with the buried depth of the soil interface. The deeper the soil interface is, the greater the energy reflection coefficient. When the interface depth of the soil is determined and the foundation is a homogeneous soil, $\gamma=0$. The greater the difference in the energy wave impedance is at the interface between the upper and lower soils, the greater $\gamma$ is.

\subsubsection{Verification Analysis by Numerical Simulation}

To verify the correctness of Eq. (6), the 1:1 equal-scale computational model of the homogeneous soil was established, and a half-sine pulse loading of $350 \mathrm{~km} / \mathrm{h}$ and a wheel-rail force of $20 \mathrm{kN}$ were used as the vibration load applying on the foundation surface for numerical analysis. Taking the maximum value of the vibration amplitude and the vibration acceleration in the time history as the normalized reference, by fitting the calculation results, the attenuation rule of the vibration acceleration along the depth of the foundation was essentially consistent with the vibration amplitude, and both satisfied the exponential function, as shown in Fig. 4. In addition, Wang et al. established the computational models to conduct the related analysis [34-36]. Zeng et al. studied the attenuation law of the vibration acceleration in the foundation under high-speed train loading through the field tests [37].

The results in this study show that the impact range of vibration acceleration generated during high-speed railway 
operation is mainly within $10 \mathrm{~m}$ of the vertical depth from the vibration source, and the vibration acceleration gradually decreases from near to far. The vibration acceleration attenuates sharply within $4 \mathrm{~m}$ from the vibration source, where the attenuation amplitude is as high as $70-80 \%$, and the attenuation rate rapidly slows down and eventually tends to zero. Thus, the attenuation curve of vibration acceleration meets the exponential distribution. The previous results verify the correctness of the theoretical derivation of the vibration acceleration attenuation from the perspectives of the numerical simulation, semianalytical model and field tests, and which indicates that the vibration acceleration can be used to represent the energy attenuation of the foundation soil instead of the amplitude.

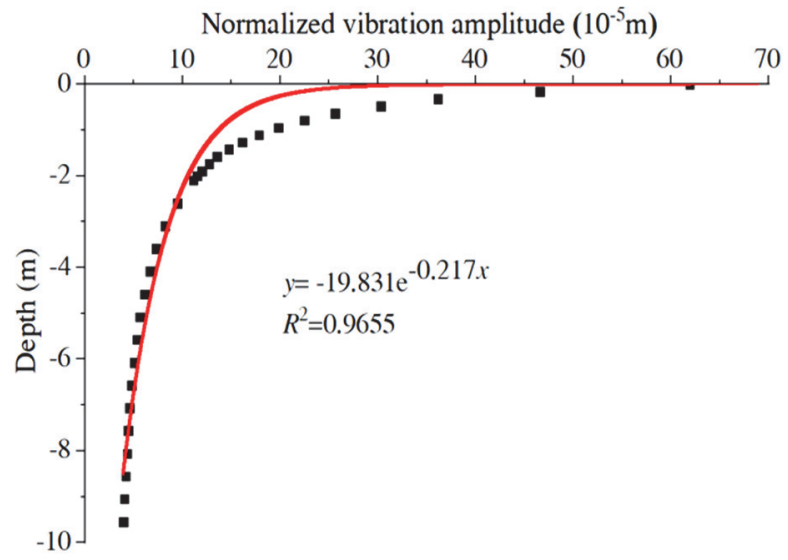

(a) Vibration amplitude

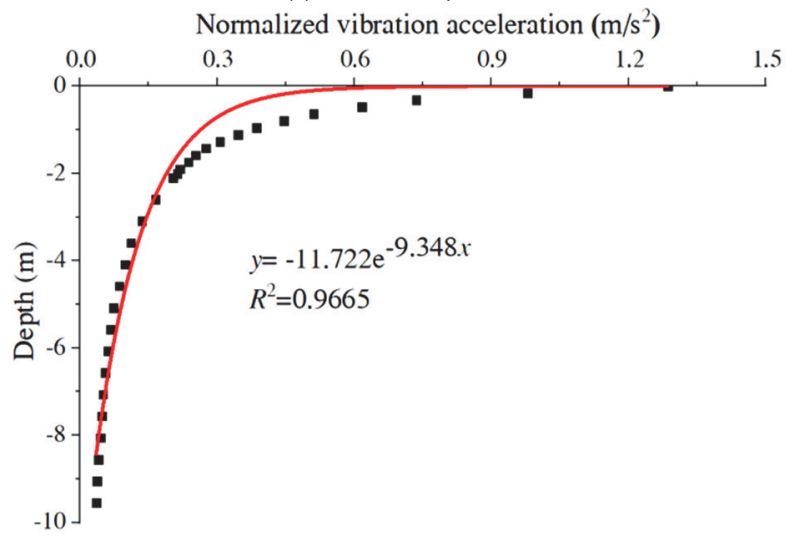

(b) Vibration acceleration

Figure 4 Attenuation curves of different variables along depth of the foundation

\subsection{Similarity Principle in the Scale Test Model}

The essential difference between the scale test model and similar test model lies in the selection of model materials, but both these models essentially reproduce the physical phenomenon similar to the full-size model. The scale test model requires that the test material be the same as the prototype test, and only the geometric dimension, external load and vibration frequency follow the similarity ratios. The similarity ratio of the corresponding physical quantity between the prototype and the scale test model is called $C$. The defined length is $L$, the displacement is $D$, the elastic module is $E$, the density is $\rho$, the stress is $\sigma$, the strain is $\varepsilon$, the cohesion is $c$, the friction angle is $\varphi$, the Poisson's ratio is $\mu$, the force is $F$, the frequency is $w$, the velocity is $v$, and the acceleration is $g$, respectively. These parameters related to the model materials in the above physical parameters are similarity ratios of 1 , such as $E, \rho$, $\sigma, \varepsilon, c$ and $\varphi$.

\subsubsection{Static Similarity Criteria}

Under the static conditions, the stress relationship of the foundation can be expressed as:

$$
f(F, L, \rho, \mu, \varphi, E, g, c)=0
$$

According to the second criterion of the similarity and dimensional analysis, the following similarity criterion was obtained by taking the length $L$, the density $\rho$, and the elastic modulus $E$ as the basic dimensions:

$$
\left\{\begin{array}{l}
\pi_{1}=\frac{\sigma}{\rho^{0} E L^{0}}, \pi_{2}=\frac{F}{\rho^{0} E L^{2}}, \pi_{3}=\frac{g}{\rho^{-1} E L^{-1}} \\
\pi_{4}=\frac{\mu}{\rho^{0} E^{0} L^{0}}, \pi_{5}=\frac{c}{\rho^{0} E L^{0}}, \pi_{6}=\frac{\varphi}{\rho^{0} E L^{0}}
\end{array}\right.
$$

The similarity constants are

$$
\left\{\begin{array}{l}
C_{F}=C_{E} C_{L}^{2}, C_{g}=C_{E} C_{\rho}^{-1} C_{L}^{-1} \\
C_{\sigma}=C_{E}=C_{C}, C_{\mu}=C_{\varphi}
\end{array}\right.
$$

\subsubsection{Dynamic SimilarityCriteria}

The dynamic characteristics of an elastic continuum can be expressed by the following equations:

$[\boldsymbol{C}]\{\boldsymbol{v}\}+[\boldsymbol{M}]\{a\}+[\boldsymbol{K}]\{\boldsymbol{u}\}=\{\boldsymbol{F}\}$

where, $\boldsymbol{C}$ is the damping matrix, $\boldsymbol{v}$ is the velocity matrix, $\boldsymbol{M}$ is the mass matrix, $\boldsymbol{a}$ is the acceleration matrix, $\boldsymbol{K}$ is the static stiffness matrix, $\boldsymbol{F}$ is the load matrix, and $\boldsymbol{u}$ is the displacement matrix.

For the dynamic problems, the function of these quantities is

$$
f(F, L, \rho, \mu, E, g, a, t, v)=0
$$

Similarly, according to the similarity criterion and the dimensional analysis, the length $L$, the density $\rho$, and the elastic modulus $E$ were taken as the basic dimensions to obtain the following similarity criterion:

$$
\left\{\begin{array}{l}
\pi_{1}=\frac{v}{\rho^{-0.5} E^{0.5} L^{0}}, \pi_{2}=\frac{g}{\rho^{-1} E L^{-1}}, \pi_{3}=\frac{\mu}{\rho^{0} E L^{0}} \\
\pi_{4}=\frac{t}{\rho^{0.5} E^{-0.5} L}, \pi_{5}=\frac{\omega}{\rho^{-0.5} E^{0.5} L^{-1}}
\end{array}\right.
$$

The similarity constants are

$$
\left\{\begin{array}{l}
C_{t}=C_{\rho}^{-0.5} C_{E}^{-0.5} C_{L}^{1}, C_{v}=C_{E}^{2} C_{\rho}^{-1} \\
C_{a}=C_{E} C_{\rho}^{-1} C_{L}^{-1}, C_{\omega}=C_{\rho}^{-0.5} C_{L}^{-1} C_{E}^{0.5}
\end{array}\right.
$$




\subsection{Numerical Calculation Analysis 3.3.1 The Computational Model}

The physical quantities are listed in Tab. 1, which was scaled according to the similarity criteria of the scale test model. The high, medium, and low vibration loads in the $1: 1$ prototype were 30,20 , and $10 \mathrm{kN}$, respectively. Five scale test models of $1: 1,1: 2,1: 5,1: 10$ and $1: 20$ were established by using Abaqus technique. Since the attenuation rate of the vibration acceleration in the foundation is very fast, the thickness of the upper soil is much smaller than that of the lower soil in the layered foundation model. Taking the prototype as an example, the thickness of the upper layer soil in the layered foundation model was $1 \mathrm{~m}$. The computational models were divided into two categories by the two different foundations: homogeneous and stratified. The Mohr-Coulomb failure criterion was adopted for the rock and soil mass, and the calculation parameters of the model are listed in Tab. 2 .

Table 1 Similarity criterion for the scale test model

\begin{tabular}{|c|c|c|c|c|}
\hline Scale ratio & Finite element model size $L \times W \times H / \mathrm{m}$ & Infinite element model size $L \times W \times H / \mathrm{m}$ & Load area $/ \mathrm{m}^{2}$ & Load / kN \\
\hline $1: 1$ & $40 \times 30 \times 20$ & $120 \times 110 \times 60$ & 1 & 20 \\
\hline $1: 2$ & $20 \times 15 \times 10$ & $60 \times 55 \times 30$ & 0.25 & 10 \\
\hline $1: 5$ & $8 \times 6 \times 4$ & $24 \times 22 \times 12$ & 0.04 & 4 \\
\hline $1: 10$ & $4 \times 3 \times 2$ & $12 \times 11 \times 6$ & 0.01 & 2 \\
\hline $1: 20$ & $2 \times 1.5 \times 1$ & $6 \times 5.5 \times 3$ & 0.0025 & 1 \\
\hline
\end{tabular}

Table 2 Physical and mechanical parameters of the model

\begin{tabular}{|c|c|c|c|c|c|c|c|}
\hline Number & Soil types & $\rho / \mathrm{kg} / \mathrm{m}^{3}$ & $c / \mathrm{kPa}$ & $\varphi /{ }^{\circ}$ & $E / \mathrm{MPa}$ & $\mu$ \\
\hline$(1)$ & Silt clay & 1704 & 12 & 19 & 32 & 0.31 \\
\hline$(2)$ & Clay & 1898 & 30 & 24 & 65 & 0.29 \\
\hline (3) & Sandy soil & 1840 & 65 & 31 & 100 & 0.03 \\
\hline
\end{tabular}

As shown in Fig. 5, to ensure the accuracy of the calculation, the grid size of the $1: 1$ model was $0.2 \mathrm{~m}$. The grid is appropriately densified at the load acting position and the interface of the soil layers, and the other grids of the model were scaled according to the corresponding ratio.

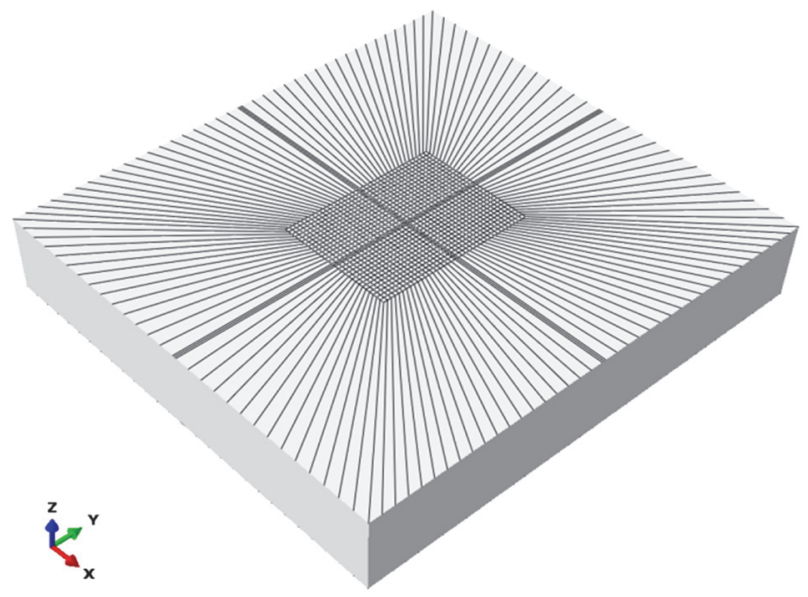

Figure $\mathbf{5}$ The computational model and its meshes

\subsubsection{Load Conditions}

Only the vertical loading action was considered, where the spatial distribution of the loading corresponded to the position of the wheel sets. Assuming that both the power vehicle and the trailer shaft weight were considered according to the maximum axle weight of the power vehicle. The pressure load was pressed on the wheel rail contact spot to represent the wheel rail interaction force, the semi-sine wave form pulse was applied:

$P(t)=P_{0} \sin ^{2}(2 \pi f t)$

where, $P$ is the pressure on the contact spot, $P_{0}$ is the maximum pressure on the contact spot, and $f$ is the frequency of the vibration.
For CRH3 train sets, the fixed wheel base of the bogie was $2.5 \mathrm{~m}$, and the vehicle fixed distance was $17.5 \mathrm{~m}$. Taking a travelling speed of $350 \mathrm{~km} / \mathrm{h}$ as an example, the frequencies of the train load were $38.89 \mathrm{~Hz}$ and $5.56 \mathrm{~Hz}$. Because the vibration of the high-speed railway infrastructure was superior to the low frequency, so the frequency of the impulse load in this study was taken as 5 $\mathrm{Hz}$, and the entire dynamic calculation time was taken as $1.0 \mathrm{~s}$, as shown in Fig. 6. According to the design specification of a high-speed railway, the frequencies of wheel-rail vibration loads caused by different train travel speeds were different. The dynamic response of the foundation soil at different train speeds was simulated by adjusting the frequency of the vibration load. The same frequency was then applied when simulating the same speed among different models. The frequencies of the vibration load under different speeds are listed in Tab. 3 .

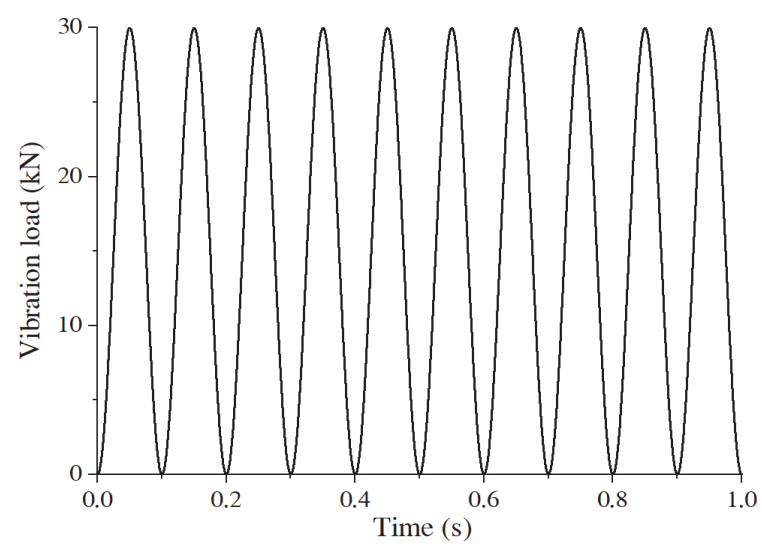

Figure 6 The time history curve of vibration load

Table 3 Vibration frequency of high-speed trains at different speeds

\begin{tabular}{|c|c|c|}
\hline \multirow{2}{*}{ Speed $/ \mathrm{km} / \mathrm{h}$} & \multicolumn{2}{|c|}{ Frequency $/ \mathrm{Hz}$} \\
\cline { 2 - 3 } & Low frequency & High frequency \\
\hline 200 & $3.18(3)$ & $22.24(22)$ \\
\hline 250 & $3.97(4)$ & $27.78(28)$ \\
\hline 300 & $4.76(4.5)$ & $33.33(33)$ \\
\hline 350 & $5.56(5)$ & $38.89(40)$ \\
\hline
\end{tabular}




\subsubsection{Boundary Conditions}

In the dynamic analysis of geoengineering, selecting boundary condition plays an important role in the accuracy and credibility of the calculation results. Additionally, to simulate the infinite foundation radiation damping is the key to the soil-structure dynamic analysis. To eliminate the influence of the static boundaries on the reflection of stress waves, the infinite element CIN3D8 was used to simulate the infinite element boundary, which could effectively simulate the radiation damping and elastic recovery performance of foundations [38].

\section{RESULTS ANALYSIS AND DISCUSSION \\ 4.1 Energy Attenuation and Size Effect Analysis of Homogeneous Foundations under Different Conditions}

The vibration load applied by the scale test model (1:5) was 6,4 and $2 \mathrm{kN}$, and the vibration frequency used to simulate the different speeds of the train was $3,4,4.5$ and $5 \mathrm{~Hz}$, respectively. The calculation results of normalized vibration acceleration under different conditions are shown in Fig. 7. The vibration acceleration propagates along the soil depth under different conditions which presents an exponential attenuation. The fitting error is within $5 \%$, which is consistent with the theoretical derivation, and the reliability of the simulation results is essentially verified. It can also be seen from Fig. 7 that the greater the driving speed and the harder the surface soil, the greater the amplitude of the initial vibration acceleration.

As seen from Fig. 7, the corresponding energy attenuation coefficient $\beta$ can be obtained through mathematical inversion. Assuming that the model scale ratio is $k$, the energy attenuation coefficient $\beta$ is taken as the ordinate and the reciprocal $1 / k$ of the model scale ratio is taken as the abscissa.

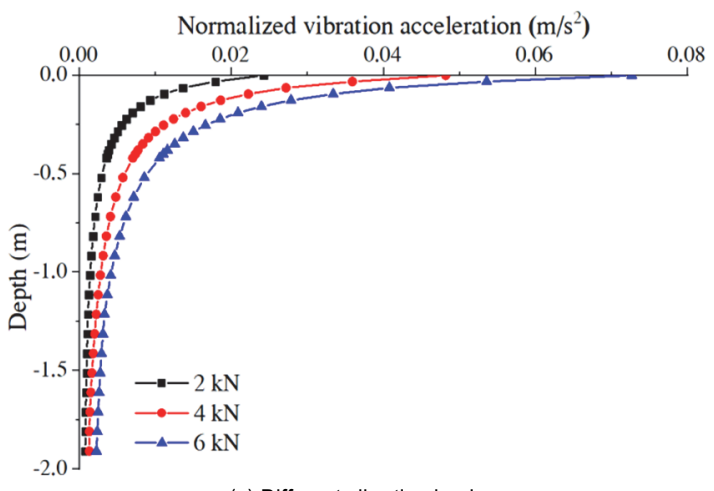

(a) Different vibration loads Normalized vibration acceleration $\left(\mathrm{m} / \mathrm{s}^{2}\right)$

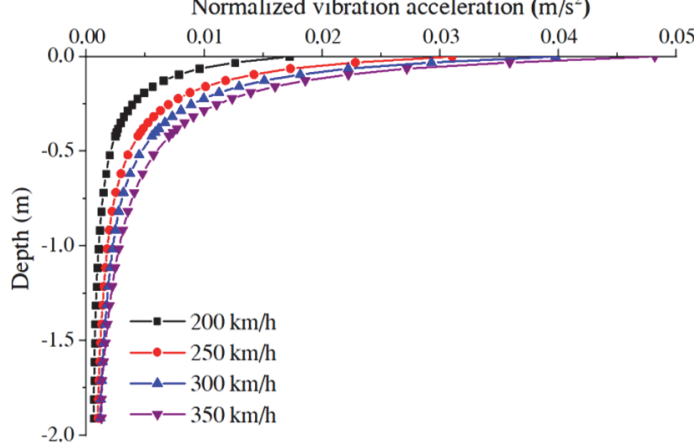

(b) Different speeds

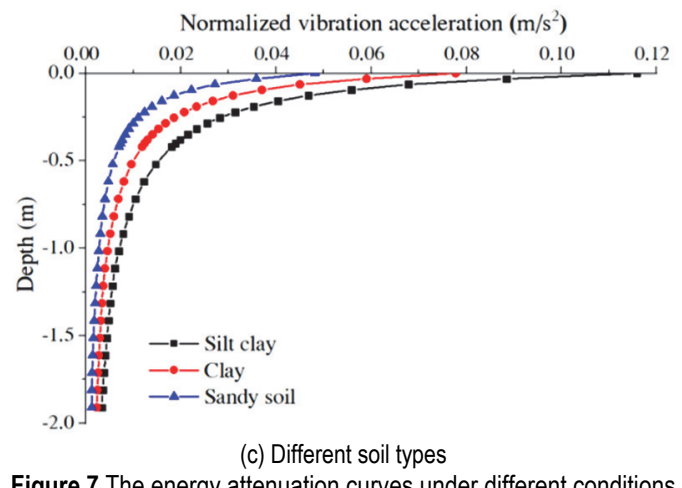

Figure 7 The energy attenuation curves under different conditions

The relationship curves of $1 / k-\beta$ are shown in Fig. 8 . As seen from Fig. 8, the worse the soil condition and the faster the train travels, the energy absorption coefficient is greater. While the vibration load is greater, the energy attenuation coefficient becomes smaller. The reciprocal $1 / k$ of the model scale ratio and the energy attenuation coefficient $\beta$ meet the exponential distribution law:

$\beta=a \mathrm{e}^{-\frac{b}{k}}+c$

where, $\beta$ is the energy attenuation coefficient, $k$ is the scale ratio, and $a, b$, and $c$ are the constants, respectively.

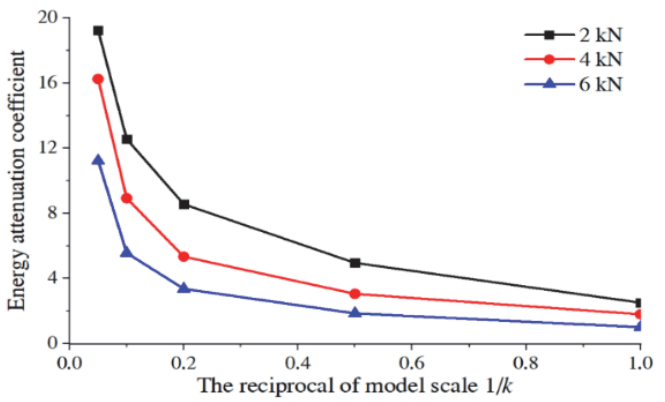

(a) Different vibration loads

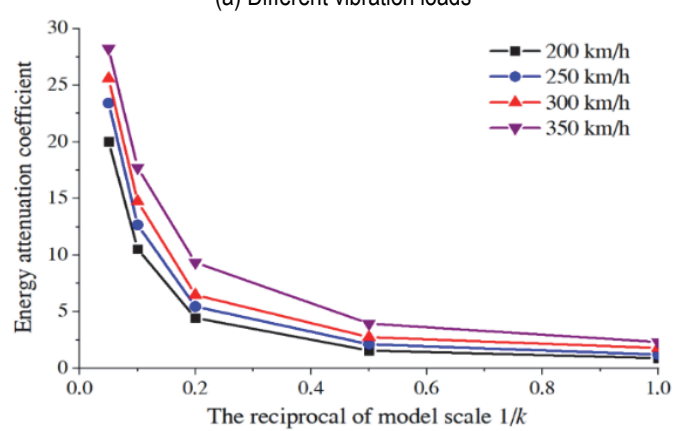

(b) Different speeds

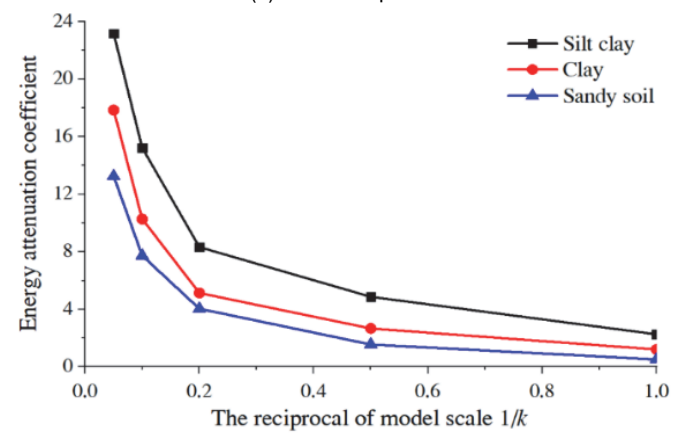

(c) Different soil types

Figure 8 The relationship curves between $\beta$ and scale ratio under different conditions 
It can be seen from Fig. 8, the driving speed and energy absorption coefficient have a positive correlation. The faster the driving speed is, the greater the energy attenuation coefficient and the faster the energy attenuation. The soil conditions and vibration loads have a negative correlation with the energy absorption coefficient. The better the soil condition and the higher the vibration load, the smaller the energy attenuation coefficient and the slower the energy attenuation trend. In addition, the larger the energy attenuation coefficient, the smaller the model scale.

As shown in Fig. 8a, the vibration load decreases from large to small, and the values of the three undetermined parameters increase correspondingly, where $a$ represents the range within the value range of $1 / k \in(0,1)$, the vibration load changes from large to small, the curve range will increase. $b$ represents the slope of the curve, the vibration load decreases from large to small, and the curve changes from gentle to steep. $c$ represents the magnitude of the upward and downward translation of the curve, where the vibration load changes from large to small, and the curve will move upward as a whole.

By calculating the parameter values range as $a(20.08$, $26.12), b(8.13,15.21)$, and $c(1.63,3.71)$, using the interpolation method can determine the values of the parameters $a, b, c$ within the range of the actual vibration load, and then let $k=1$, then the energy attenuation coefficient $\beta$ of the foundation prototype can be obtained. Substituting $\beta$ into Eq. (13), the vibration acceleration amplitude at depth $H$ of the foundation can be predicted. Similarly, the vibration acceleration amplitude of the prototype under other variables can be predicted.

\subsection{Energy Reflection and Size Effect Analysis at the Interface of Layered Foundations}

Taking 1:5 model as an example, the interface of the soil was located $0.2 \mathrm{~m}$ away from the ground surface, where the vertical attenuation of the vibration acceleration is shown in Fig. 9. The acceleration attenuation curve has a turning point at the interface of the soil in the layered foundation.

It no longer propagates according to the attenuation law of the homogeneous soil, the interface of the soil is at the boundary. Above the interface follows the attenuation law of the upper soil, and below the interface follows the attenuation law of the lower soil. Eq. (10) shows that the energy reflection coefficient of the interface is related not only to the energy attenuation coefficient of the upper and lower layers, but also to the thickness of the upper layer. Since the energy attenuation coefficient is related to the model scale, it can be inferred that the energy reflection coefficient of the interface is also closely related to the reduced scale.

Fig. 10 shows the energy reflection coefficient $\gamma=0$ in the homogeneous foundation. The greater the difference between the upper and lower soil quality of the soil layer, the greater the energy reflection coefficient. The energy reflection coefficient at the interface decreases as the scale of the model decreases, and the rate of the reflection coefficient decrease also slows down. The fitting effect of the quadratic function is determined to be the best, the fitting error is less than $1 \%$, and the size effect formula of the interface energy reflection coefficient is obtained as:

$\gamma=p\left(\frac{1}{k}\right)^{2}+q\left(\frac{1}{k}\right)+r$

where, $p, q$ and $r$ are the constants, and $k$ is the scale ratio.
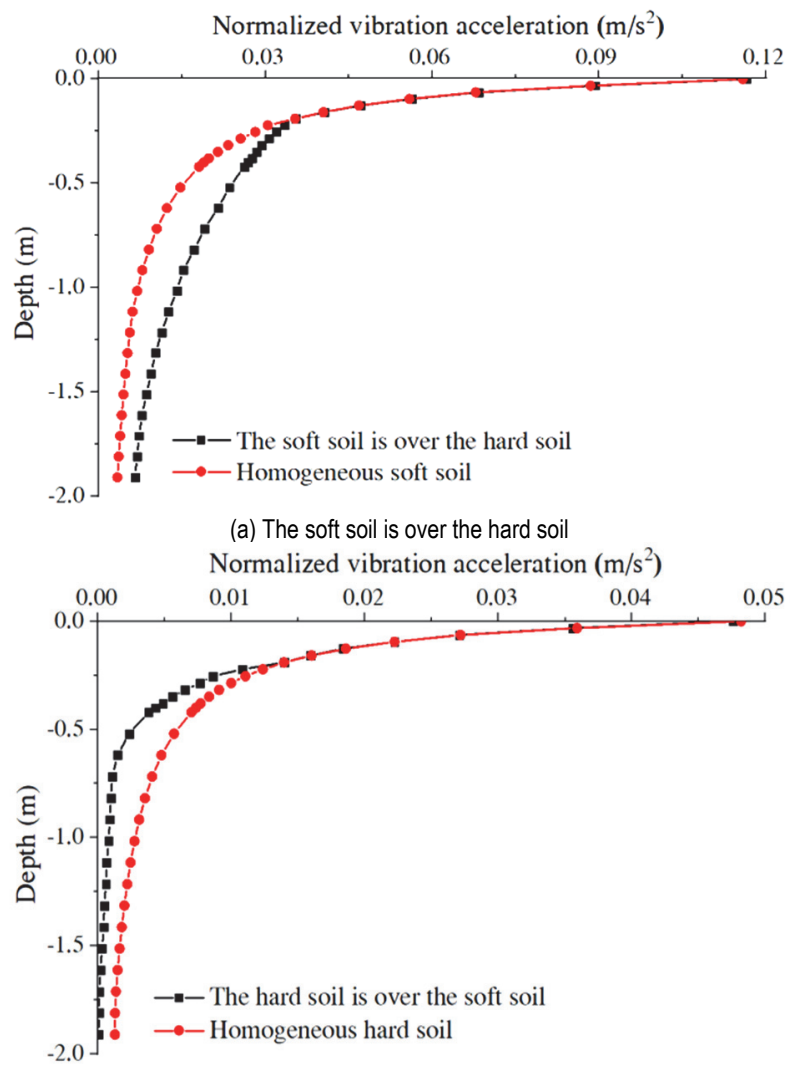

(b) The hard soil is over the soft soil

Figure 9 The acceleration attenuation curves of the layered foundation.

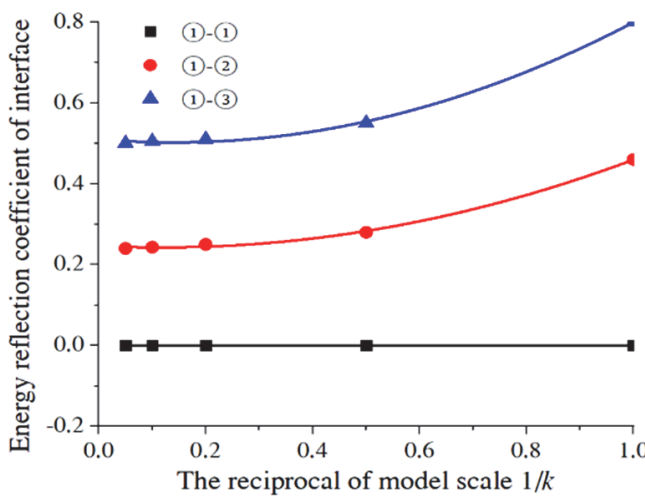

Figure 10 The relationship curves between the energy reflection coefficient and the scale ratio

The difference of the soil properties between the upper and lower layers of the foundation changes from small to large, and the values of the three undetermined parameters will increase accordingly. According to the method in Section 4.1, the values of parameters $p, q$, and $r$ are determined by the interpolation method at the interface of the foundation. Let $k=1$ and substitute it into Eq. (20), the energy reflection coefficient at the interface of depth $H$ can be predicted. At the same time, three sets of $\beta_{1}, \beta_{2}$ can be obtained by three scale test models with different similarity 
ratios. Combining Eqs. (10) and (20), the accuracy values of $p, q$ and $r$ can be obtained. Letting $k=1$ and substituting it into Eq. (20), the energy reflection coefficient at the interface of the prototype model can also be obtained.

\subsection{Verification and Analysis of Indoor Model Test}

Considering the laboratory and economic conditions, the independently designed and manufactured model device was adopted in the indoor model test $[39,40]$. The sizes of the model box were Length $\times$ Width $\times$ Height $=$ $1.2 \times 0.8 \times 0.6 \mathrm{~m}$. As shown in Fig. 11 , the small electric vibration exciter DH40500 was used as the loading device, the frequency sweep signal generator DH1301 and the dynamic data acquisition instrument DH5923 were used in the study. As the vibration load of the scale model is reduced, the vibration acceleration generated by the vibration load in the foundation will be correspondingly reduced. Therefore, the high-sensitivity capacitive acceleration sensor was adopted to collect the data.
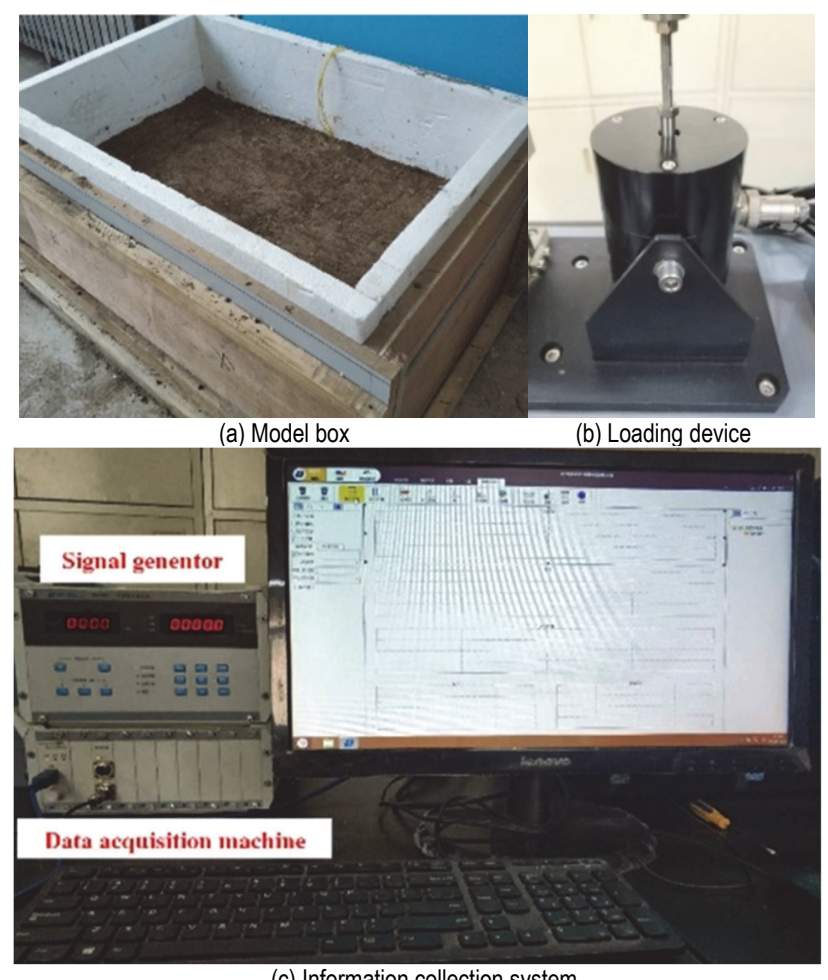

(c) Information collection system

Figure 11 Test equipment of the model

Three sets of laboratory model tests with scale ratios of 1:50,1:75, and 1:100 were conducted and each set of similarity ratios also included three model tests of silt clay, clay and sandy soil. The soil in the model box was consistent with the undisturbed soil. To eliminate the boundary effect and stress wave reflection of the model box before laying the soil layer, the $100 \mathrm{~mm}$ polystyrene plastic board was installed inside the device [41, 42]. Before the soil was laid, a layer of petroleum jelly was applied on the sidewall of the plastic board to reduce the friction between the soil and the sidewall of the plastic board. The soil layer should be laid in the layered and compacted type. The acceleration sensor was buried every $4 \mathrm{~cm}$ under the dynamic load position to monitor the data in the foundation. After the model was finished, the model box was sealed and placed, and the vibration test was conducted after the soil was naturally consolidated.

As shown in Fig. 12, the harder the soil under the same scale and vibration load conditions, the greater the initial vibration acceleration and the faster the attenuation rate. As the depth increases, the vibration acceleration decreases gradually and the attenuation rate becomes slower. The energy attenuation coefficients of the experimental model are listed in Tab. 4.

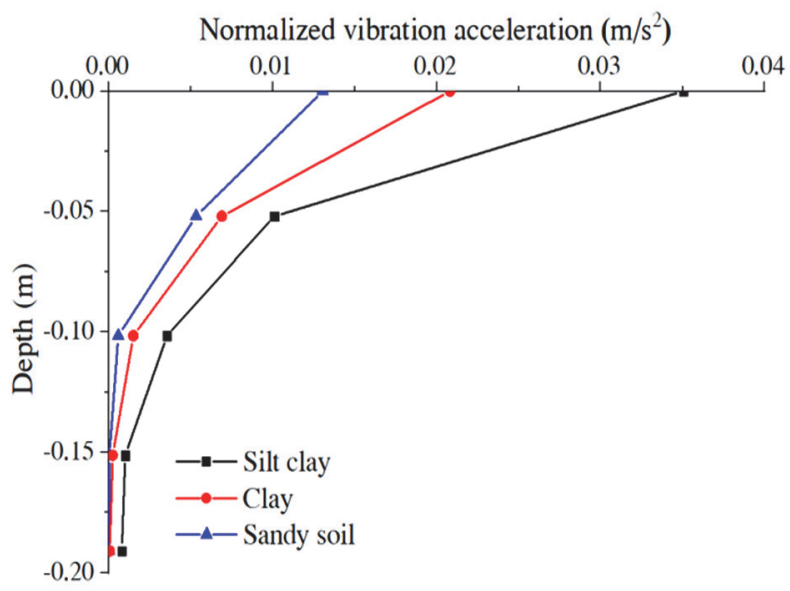

(a) $1: 50$

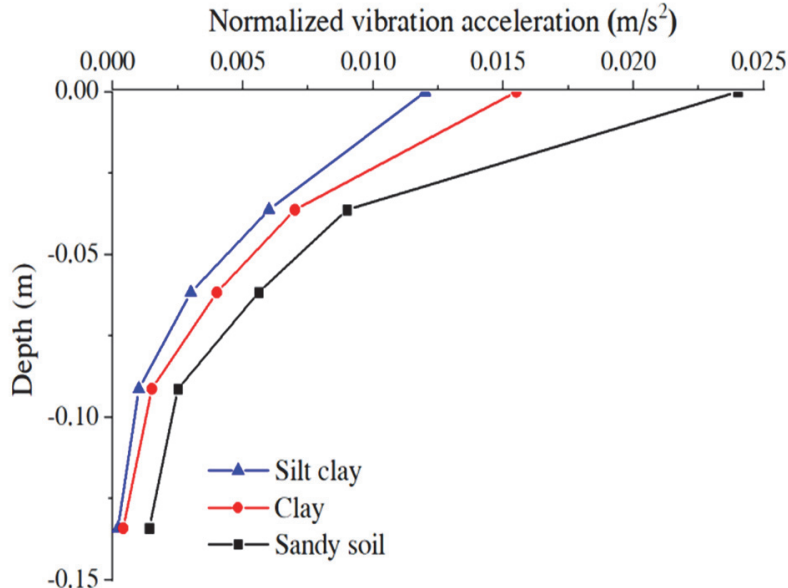

(b) 1:75

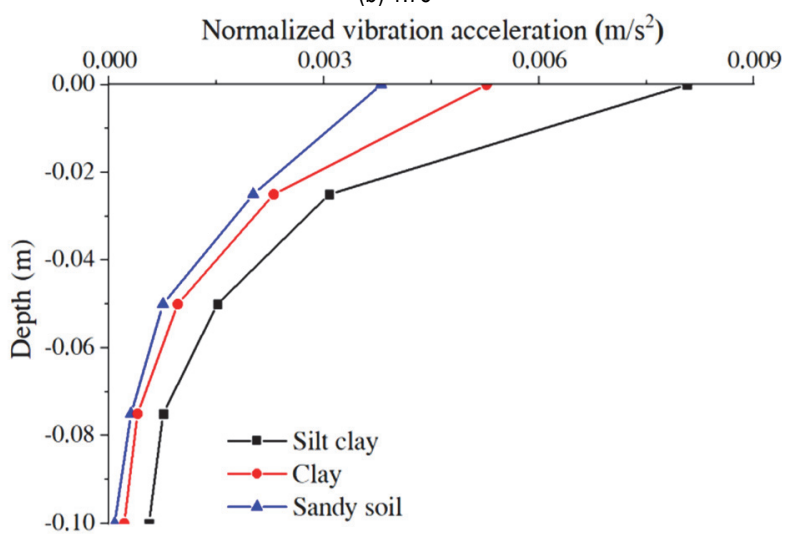

(c) 1:100

Figure 12 Variation curves of indoor model test with different scale ratios

Table 4 Energy attenuation coefficient of different scale models

\begin{tabular}{|c|c|c|c|}
\hline \multirow{2}{*}{$\begin{array}{c}\text { The energy attenuation } \\
\text { coefficients }\end{array}$} & \multicolumn{3}{|c|}{ Scale ratio } \\
\cline { 2 - 4 } & $1: 50$ & $1: 75$ & $1: 100$ \\
\hline$\beta_{1}$ & 24.341 & 28.316 & 32.315 \\
\hline$\beta_{2}$ & 23.183 & 24.852 & 31.052 \\
\hline$\beta_{3}$ & 21.156 & 23.284 & 26.286 \\
\hline
\end{tabular}


The relationship among the vibration load, the reciprocal of the model scale, and the energy attenuation coefficient can be expressed nonlinearly, and the relationship among the vibration frequency, the reciprocal of the model scale, and the energy attenuation coefficient is the same.

Then, the two-factor relationship equation for the energy attenuation coefficient of the foundation as a function of the reciprocal of the model scale, the vibration load (or the frequency) is constructed as:

$$
\beta=\left(A \mathrm{e}^{-B y}+C\right)\left(D \mathrm{e}^{-E x}+F\right)
$$

where, $A, B, C, D, E$ and $F$ are the constants, which can be obtained via least-squares fitting, $y$ is the reciprocal of the scale of the model, and $x$ is the vibration load or the frequency.

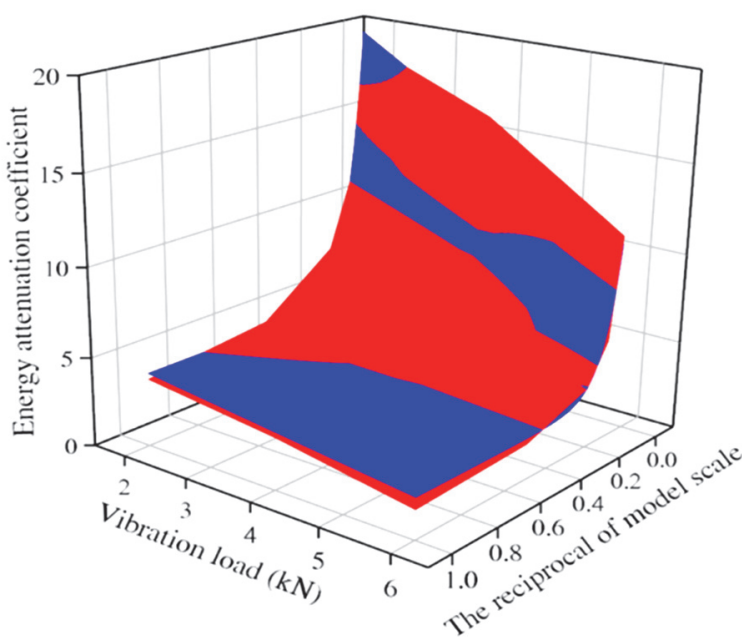

(a) Different loads

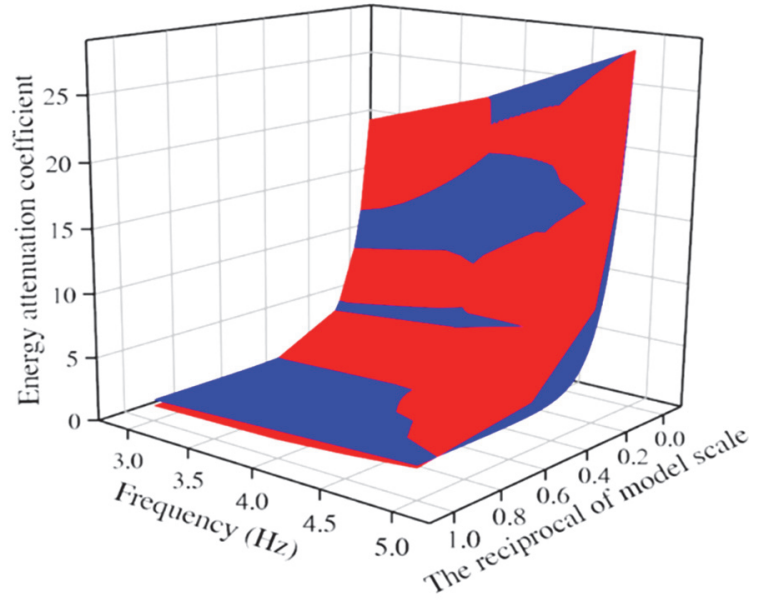

(b) Different frequencies

Figure 13 Three-dimension relationships between the model scale and energy attenuation coefficient under different conditions

Taking the sand soil as an example, based on the indoor model tests (1:50, 1:75 and 1:100), the calculation models of homogeneous subgrades were constructed. As shown in Fig. 13, the corresponding data on the load, the frequency, the reciprocal of the model scale and the energy attenuation coefficient are plotted in three-dimension coordinates. The red surface is composed of the experimental data, and the blue one is composed of the numerical analysis results. The two sets of data have a high coincidence degree, which essentially verifies the reliability of the simulation results. The experimental and the simulation data are fitted by Eq. (21). The values of parameters in Eq. (21) are listed in Tab. 5. The correlation coefficients are 0.977 and 0.989 , respectively, which proves that the equation is reasonable.

The energy attenuation coefficients $\beta_{1}$ and $\beta_{3}$ of sandy soil and silty clay, respectively, were selected from Tab. 4 . The related data being substituted into Eq. (20), then $p, q$, and $r$ is $0.2,0.09$, and 0.463, respectively, and the size effect formula of the energy reflection coefficient is obtained. Letting $k=1$, the energy reflection coefficient of the prototype soil is 0.753 , and the errors among the predicted value, the numerical and theoretical analysis are less than $6 \%$, which verifies the reliability of the size effect formula proposed based on the energy attenuation.

Table 5 Fitting parameters
\begin{tabular}{|c|c|c|c|c|c|c|c|}
\hline Name & $A$ & $B$ & $C$ & $D$ & $E$ & $F$ & $R^{2}$ \\
\hline Load & 42.8 & 317.2 & 13.6 & -5.0 & 5.1 & 0.1 & 0.977 \\
\hline Frequency & 27.0 & 487.3 & 11.6 & 3.9 & -3.8 & 0.3 & 0.989 \\
\hline
\end{tabular}

Accurately determining the energy attenuation and the energy reflection coefficients in the soil layer is the key factor in analyzing the dynamic response characteristics of the foundation, which plays a vital role in predicting the cumulative deformation of the foundation under the highspeed train loading. In practical engineering, it is difficult to obtain the energy attenuation coefficient of the foundation and the reflection coefficient at the interface by means of field tests. Scale model tests can greatly save manpower and material resources. According to the size effect formula proposed in this study, the energy attenuation and the interface energy reflection coefficients can be determined more accurately and quickly, which can play an important role in similar engineering.

\section{CONCLUSION}

To investigate the dynamic size effect of the scale test model of the foundation under the high-speed railway dynamic loading, the theoretical analysis, numerical simulation, and indoor model tests were conducted. The main conclusions are as follows:

(1) The energy attenuation coefficient can characterize the energy attenuation in the foundation, which is closely related to the soil type, the load amplitude, and the frequency. To study the size effect of the scale test model is feasible in view of dynamics based on the energy absorption characteristics of soil. The relationship between the energy attenuation coefficient and the scale of the test model is constructed, which reveals the size effect of the energy attenuation in the homogeneous foundation.

(2) The size effect formula is proposed by which the vibration acceleration at different depths can be predicted in the foundation. The vibration acceleration propagation in the layered soil has different attenuation rates, which increase with increase of the wave impedance. At the interface of the soil layer, the vibration acceleration will appear at the inflection point and will continue transferring according to the attenuation rule of the subsoil. The energy reflection coefficient at the interface in the layered foundation is proposed. 
(3) With the help of scale model tests and the mathematical inversion, the energy reflection coefficient of the soil layer interface in a prototype foundation can be predicted. The energy attenuation coefficient is directly proportional to the vibration frequency and inversely proportional to the load. The two-factor equation of the energy attenuation coefficient is established based on the nonlinear relationship among the energy attenuation coefficient, the model scale, the load amplitude, and the vibration frequency.

The size effect response equation of the scale test model derived in this study can be mainly used early and the mid-term of the high-speed railway operation. In the later stage of that, the mechanical properties of the foundation will inevitably deteriorate, and more obvious plastic deformation will simultaneously occur. Therefore, the size effect of the scale test model of the foundation in the elastoplastic state will be further studied in the future.

\section{Acknowledgements}

This study was financially supported by the National Natural Science Foundation of China (U1810203), and the Fundamental Research Funds for the Universities of Henan Province (NSFRF200202), China.

\section{REFERENCES}

[1] Rahpeyma, S., Halldorsson, B., \& Hrafnkelsson, B. (2019). Site effect estimation on two Icelandic strong-motion arrays using a Bayesian hierarchical model for the spatial distribution of earthquake peak ground acceleration. Soil Dynamics and Earthquake Engineering, 120, 369-385. https://doi.org/10.1016/.j.soildyn.2019.02.007

[2] Ma, K. K., Li, B., \& Wang, D. (2019). Experimental study and numerical analysis on ground vibration characteristics of subgrades section of Baoji-Lanzhou high-speed railway. Journal of Railway Science and Engineering, 16(2), 294301. https://doi.org/10.19713/j.cnki.43-1423/u.2019.02.003

[3] Feng, H. X. \& Yi, W. J. (2020). Experimental study on shear performance and bearing capacity of prestressed concrete Tbeams. Dyna, 95(4), 405-411. https://doi.org/10.6036/9655

[4] Wen, X. G., Hu, P. C., \& Zhou, J. (2014). Experimental research on influence of cracks on size effect of electroosmosis model. Chinese Journal of Geotechnical Engineering, 36(11), 2054-2060. https://doi.org/10.11779/CJGE201411011

[5] Shahu, J. T. \& Reddy, Y. R. (2012). Clayey soil reinforced with stone column group: model tests and analyses. Journal of Geotechnical and Geoenvironmental Engineering, 137(12), 1265-1274 https://doi.org/10.1061/(asce)gt.1943-5606. 0000552

[6] Li, D., Li, Y., \& Zhu, W. (2020). Analytical modelling of load-displacement performance of cable bolts incorpatrating crack propagation. Rock Mechanics and Rock Engineering, 53, 3471-3483. https://doi.org/10.1007/s00603-020-02123-1

[7] Jayasree, P. K., Rajagopal, K., \& Gnanendran, C. T. (2012). Influence of sidewall friction on the results of small-scale laboratory model tests: numerical assessment. International Journal of Geomechanics, 12(2), 119-126. https://doi.org/10.1061/(asce) gm.1943-5622.0000120

[8] Togun, N. \& Bağdatli, S. M. (2018). The vibration of nanobeam resting on elastic foundation using modified couple stress theory. Technical Journal, 12(4), 221-225. https://doi.org/10.31803/tg-20180214212115

[9] Gora, J. \& Szafraniec, M.(2020). Influence of maximum aggregate grain size on the strength properties and modulus of elasticity of concrete. Applied Sciences-basel, 10(11), 113. https://doi.org/10.3390/app10113918

[10] Li, D., Masoumi, H., Li, Y., Hagan, P., Saydam, S., \& Asadizadeh, M. (2020). Assessing the mechanical performance of different cable bolts based on design of experiments techniques and analysis of variance. International Journal of Rock Mechanics and Mining Science, 130, 1-12. https://doi.org/10.1016/j.jijmms.2020.104307

[11] Jin, L., Yu, W. X., \& Du, X. L. (2020). Meso-scale simulations of size effect on concrete dynamic splitting tensile strength: Influence of aggregate content and maximum aggregate size. Engineering Fracture Mechanics, 230, 1-18. https://doi.org/10.1016/j.engfracmech.2020.106979

[12] Du, M., Jin, L., \& Li, D. (2017). Mesoscopic simulation study of the influence of aggregate size on mechanical properties and specimen size effect of concrete subjected to splitting tensile loading. Engineering Mechanics, 34(9), 5463. https://doi.org/10.6052/j.issn.1000-4750.2016.02.0122

[13] Yu, F., Sun, D. Q., \& Wang, J. (2019). Influence of aggregate size on compressive strength of pervious concrete. Construction and Building Materials, 209, 463-475. https://doi.org/10.1016/j.conbuildmat.2019.03.140

[14] Knox, C. L., Dizhur, D., \& Ingham, J. M. (2018). Experimental study on scale effects in clay brick masonry prisms and wall panels investigating compression and shear related properties. Construction and Building Materials, 163, 706-713. https://doi.org/10.1016/j.conbuildmat.2017.12.149

[15] Pirooznia, A. \& Moradloo, A. (2020). Investigation of size effect and smeared crack models in ordinary and dam concrete fracture tests. Engineering Fracture Mechanics, 262, 1-25. https://doi.org/10.1016/j.engfracmech.2019.106863

[16] Gao, X. F., Koval, G., \& Chazallon, C. (2016). A size and boundary effects model for quasi-brittle fracture. Materials, 9(12), 1-20. https://doi.org/10.3390/ma9121030

[17] Alam, S. Y., Zhu, R., \& Loukili, A. (2020). A new way to analyse the size effect in quasi-brittle materials by scaling the heterogeneity size. Engineering Fracture Mechanics, 225, 113. https://doi.org/10.1016/j.engfracmech.2019.106864

[18] Chen, J. L., Dong, Y. P., \& Whittle, A. J. (2020). Prediction and evaluation of size effects for surface foundations on sand. Journal of Geotechnical and Geoenvironmental Engineering, 146(5), 1-13 https://doi.org/10.1061/(ASCE)GT. 1943-5606.0002237

[19] Jin, L., Li, P., \& Du, X. L. (2020). Size effect on nominal strength of circular stirrup-confined RC columns under axial compression: mesoscale study. Journal of Structural Engineering, 146(3), 1-13. https://doi.org/10.1061/(ASCE)ST. 1943-541X.0002516

[20] Liu, P., Yang, H. G., \& Fan, Z. (2016). Experimental study on scale effect of rigid pile composite foundation. Chinese Journal of Rock Mechanics and Engineering, 35(1), 187200. https://doi.org/10.13722/j.cnki.jrme.2014.1257

[21] Yoder, M., Thompson, L., \& Summers, J. (2019). Size effects in lattice-structured cellular materials: edge softening effects. Journal of Materials Science, 54(5), 3942-3959. https://doi.org/10.1007/s10853-018-3103-9

[22] Lee, S. \& Han, J. T. (2015). Numerical analysis on the size effect of a footing. Journal of Korea Academia-industrial Cooperation Society, 16(1), 778-784. https://doi.org/10.5762/KAIS.2015.16.1.778

[23] Vishwakarma, R. J. \& Ingle, R. K. (2018). Effect of panel size and radius of relative stiffness on critical stresses in concrete pavement. Arabian Journal for Science and Engineering, 43(10), 5677-5687. https://doi.org/10.1007/s13369-018- 3308-x

[24] Mehrjardi, G. T. \& Khazaei, M. (2017). Scale effect on the behaviour of geogrid-reinforced soil under repeated loads. Geotextiles and Geomembranes, 45(6), 603-615. https://doi.org/10.1016/j.geotexmem.2017.08.002

[25] Krauthammer, T., Elfahal, M. M., \& Lim, J. (2003). Size 
effect for high-strength concrete cylinders subjected to axial impact. International Journal of Impact Engineering, 28(9), 1001-1016. https://doi.org/10.1016/S0734-743X(02)00166-5

[26] Wang, S. R., Zhang, J. Y., Li, J. T., Kong, F. L., \& Fan, J. Q. (2020). Analysis of vibration attenuation and energy consumption of blasting demolition chimney. Tehnicki Vjesnik - Technical Gazette, 27(3), 826-834. https://doi.org/10.17559/TV-20200225022727

[27] Song, X. R., Huang, C. L., Zhang, G. F., Shan, W. C., \& Wang, Y. M. (2019). Experimental study on seismic performance of new-type composite shear wall. Dyna, 94(4), 465-472. https://doi.org/10.6036/9192

[28] Wang, S. R., Shi, K. P., He, Y. S., \& Wang, X. Q. (2019). Dynamic response analysis of middle pillar for ultra-small spacing tunnels under train vibration loads. Journal of Engineering Science and Technology Review, 12(3), 30-37. https://doi.org/10.25103/jestr.123.05

[29] Kim, K. M., Lee, S., \& Cho, J. Y. (2019). Effect of maximum coarse aggregate size on dynamic compressive strength of high-strength concrete. International Journal of Impact Engineering, 125, 107-116. https://doi.org/10.1016/j.ijimpeng.2018.11.003

[30] He, Q., Li, Y., Li, D., \& Zhang, C. (2020). Microcrack fracturing of coal specimens under quasi-static combined compression and shear loading. Journal of rock mechanics and geotechnical engineering, 12(5), 1014-1026. https://doi.org/10.1016/j.jrmge.2020.01.009

[31] Jin, L., Yu, W. X., \& Du, X. L. (2019). Dynamic size effect of concrete under tension: a numerical study. International Journal of Impact Engineering, 132, 1-13. https://doi.org/10.1016/j.ijimpeng.2019.103318

[32] Lee, B. J., Kee, S. H., \& Oh, T. (2015). Effect of cylinder size on the modulus of elasticity and compressive strength of concrete from static and dynamic tests. Advances in Materials Science and Engineering, 2015, 1-12. https://doi.org/10.1155/2015/580638

[33] Wang, X. H., Zhang, S. R., \& Wang, C. (2018). Fragmentation-based dynamic size effect of layered roller compacted concrete (RCC) under impact loadings. Construction and Building Materials, 192, 58-69. https://doi.org/10.1016/j.conbuildmat. 2018.10.161

[34] Wang, S. R., Shi, K. P., \& Li, Z. H. (2019). Spatial distribution law of vibration acceleration of ultra-smallspacing tunnel under train moving loads. Journal of Engineering Science and Technology Review, 12(6), 96-104. https://doi.org/10.25103/jestr.126.12

[35] Cilingir, U. \& Madabhushi, S. P. G. (2011). A model study on the effects of input motion on the seismic behavior of tunnels. Soil Dynamics and Earthquake Engineering, 31(3), 452-465. https://doi.org/10.1016/j.soildyn.2010.10.004

[36] Pisačić, K., Horvat, M., \& Botak, Z. (2019). Finite difference solution of plate bending using Wolfram Mathematica. Technical Journal, 13(3), 241-247. https://doi.org/10.31803/tg-20190328111708

[37] Zeng, Z., Wang, J., \& Yin, H. (2019). Experimental investigation on the vibration reduction characteristics of an optimized heavy-haul railway low-vibration track. Shock and Vibration, 2019, 1-17. https://doi.org/10.1155/2019/1539564

[38] Yi, H. Y., Qi, T. Y., \& Qian, W. P. (2019). Influence of longterm dynamic load induced by high-speed trains on the accumulative deformation of shallow buried tunnel linings. Tunneling and Underground Space Technology, 84, 166-176. https://doi.org/10.1016/j.tust.2018.11.005

[39] Li, Y. C., Wang, S. R., \& He, Y. S. (2020). Multi-objective optimization of construction project based on improved ant colony algorithm. Tehnicki Vjesnik - Technical Gazette, 27(1), 184-190. https://doi.org/10.17559/TV-20191212113720

[40] Li, D., Masoumi, H., Li, Y., Hagan, P., Saydam, S., \& Asadizadeh, M. (2020). Assessing the mechanical performance of different cable bolts based on design of experiments techniques and analysis of variance. International Journal of Rock Mechanics and Mining Science, 130, 1-12. https://doi.org/10.1016/i.jijmms.2020.104307

[41] Ulas, C. (2011). A model study on the effects of input motion on the seismic behavior of tunnels. Soil Dynamics and Earthquake Engineering, 31(3), 452-465. https://doi.org/10.1016/j.soildyn.2010.10.004

[42] Mariaca-Beltran, Y. D., Garcia-Salmoran, I. A., ClementeMirafuente, C. M., Rodriguez-Ramirez, J. A., GarciaCastrejon, J. C., \& Acosta-Flores, M. (2019). New methodology for the analysis of mechanical system using scale models and similarity laws. Dyna, 94(1), 59-66. https://doi.org/10.6036/8825

\section{Contact information:}

\section{Shuren WANG, PhD, Professor}

(Corresponding author)

1) International Joint Research Laboratory of Henan Province for Underground Space Development and Disaster Prevention, Henan Polytechnic University

2) State Collaborative Innovation Center of Coal Work Safety and Clean-efficiency Utilization, Henan Polytechnic University,

No. 2001 Century Avenue, Jiaozuo, Henan Province, 454003, China

E-mail:w_sr88@163.com

\section{Kunpeng SHI, PhD, Candidate}

School of Civil Engineering, Henan Polytechnic University,

No. 2001 Century Avenue, Jiaozuo, Henan Province, 454003, China

E-mail: 444583054@qq.com

Youfeng ZOU, PhD, Professor

International Joint Research Laboratory of Henan Province for Underground Space Development and Disaster Prevention, Henan Polytechnic University, No. 2001 Century Avenue, Jiaozuo, Henan Province, 454003, China

E-mail: zouyf@hpu.edu.cn

\section{Zhengsheng ZOU, PhD, Professo}

International Joint Research Laboratory of Henan Province for Underground Space Development and Disaster Prevention, Henan Polytechnic University, No. 2001 Century Avenue, Jiaozuo, Henan Province, 454003, China E-mail: zouzs@hpu.edu.cn

Xuchun WANG, PhD, Professor

School of Civil Engineering, Qingdao University of Technology,

No. 11 Fushun Road, Qingdao, Shandong Province, 266033, China

E-mail: xuchun_wang@263.net

Chunliu LI, PhD, Associate Professor

School of Urban Construction,

Hebei Normal University of Science and Technology,

No. 360 Hebei West Street, Qingdao, Hebei Province, 066004, China

E-mail: Iclcc_010@163.com 